\title{
Perceived exertion influences pacing among ultramara- thon runners but post-race mood change is associated with performance expectancy
}

\author{
Dominic Micklewright (PhD) \\ Eleni Papadopoulou (BSc) \\ David Parry (MSc) \\ University of Essex, United Kingdom \\ Tamara Hew-Butler (PhD) \\ Nicholas Tam (BSc) \\ Timothy Noakes (DSc) \\ University of Cape Town, South Africa
}

\begin{abstract}
Objectives. This study investigated whether post-race mood changes among ultramarathon runners are associated with perceived exertion or the discrepancy between their actual and predicted performance times.

Methods. Eight runners completed the Puffer ultramarathon, which is a challenging $73 \mathrm{~km}$ mountainous race across Table Mountain National Park in South Africa. Each runner completed a series of profile of mood state questionnaires (POMS) 2 days before the race (baseline), on the morning of the race (pre-race) and immediately after the race (post-race). Ratings of perceived exertion (RPE) were measured at 13 points during the race using the Borg 6-20 scale. The accuracy of performance expectations was measured as the difference between runners' actual and predicted race times.

Results. Average completion time was 11:31:36 $\pm 00: 26: 32$ (hh: $\mathrm{mm}: \mathrm{ss}$ ), average running speed was $6.4 \pm 2.2 \mathrm{~km} \cdot \mathrm{hr}^{-1}$ and average RPE was 14.1 \pm 2.0 . Increased POMS confusion was found before the race (33.30.7 v. $37.1 \pm 5.2, p=0.014$; baseline v. pre-race). Post-race increases in POMS total mood disturbance (TMD) were
\end{abstract}

\section{CORRESPONDENCE:}

Dr Dominic Micklewright

Centre for Sports and Exercise Science

Department of Biological Sciences

University of Essex

Wivenhoe Park

Colchester

Essex

UK, CO4 3SQ

E-mail: dpmick@essex.ac.uk found $(168.3 \pm 20$ v. $137.5 \pm 6.3, p=0.001$; post race v. baseline) characterised by decreased vigour $(43.3 \pm 4.0$ v. $33.5 \pm 7.0$, $p=0.008$; baseline $\mathrm{v}$. post race), increased confusion $(33.3 \pm 0.7 \mathrm{v}$. $38.5 \pm 4.8, p=0.006$; baseline $v$. post race) and increased fatigue (37.8 \pm 4.8 v. $53.8 \pm 7.3, p=0.0003$; baseline v. post race). A linear increase in RPE was found during the race $(r=0.737, p=0.002)$ The magnitude of their post-race mood change ( $r=-0.704$ $p=0.026$ ) was not found to be associated with runners' average RPE but was found to be negatively correlated with accuracy of the performance predictions. A time series analysis indicated that POMS TMD would have taken $142 \pm 89 \mathrm{~min}$ to recover.

Conclusions. The results show that RPE influences the way ultramarathon runners pace themselves more than performance expectancy but performance expectations have a greater influence on post-race mood. The magnitude of post-race mood change is associated with the extent of discrepancy between runners' predicted and actual performance. This has implications for designing appropriate goals and pacing strategies for ultraendurance athletes.

\section{Introduction}

An ultramarathon is an endurance running event that can vary in duration from $50 \mathrm{~km}$ during a single day to in excess of $200 \mathrm{~km}$ over several days. The physiological and immunological consequences of ultramarathon running have been fairly well documented, highlighting the demanding nature of this type of event. ${ }^{1-4}$ The physiological demands associated with ultramarathon running potentially have psychological consequences yet there is relatively little coverage of this in the literature. Of the studies which have been published, most have focused on personality, ${ }^{5}$ perceived exertion ${ }^{6}$ and coping ${ }^{7}$ but none have specifically explored the relationship between mood and ultramarathon performance.

Several studies have reported positive effects of running on mood including reduced feelings of depression and confusion. ${ }^{8-10}$ Even though neutral or negative emotional responses to running 
have been reported in a few studies, ${ }^{11,12}$ the generally accepted conclusion is that running is good for mental health. ${ }^{13}$ The positive psychological changes associated with recreational running probably only have a limited amount of relevance to the much more prolonged and demanding nature of ultramarathon running yet, as the lack of ultramarathon literature shows, this is an under-researched area of sport psychology. In one of the few studies available, a complex pattern of mood change among ultramarathon finishers was found to include reduced tension and vigour with increased fatigue, depression and confusion. ${ }^{14}$ What their results suggest is an association between ultra-endurance running and unpleasant mood state characterised by changes in the high activation states of tension and vigour, as well changes in the low activation states of fatigue, depression and confusion. Interestingly, they account for the change in tension as relief from pre-race anxiety, and the changes in vigour, fatigue, depression and confusion as due to the unique sensations associated with running for such a long time. Their conclusions are valid but what needs further consideration is perhaps how intra-individual differences in runners' circumstances, motives, perceptions of the race environment and conscious cognitive processes potentially influence the way endurance running sensations are interpreted by a runner as an antecedent of perceived exertion and affective experience.

According to cognitive theories of emotion the relationship between an event and an emotional response is mediated by various forms of conscious information processing. Several theories, such as attribution theory ${ }^{15}$ and appraisal theory, ${ }^{16}$ can help to explain how an individual's conscious thoughts can shape their emotional experience associated with ultra-endurance running. A claim often made is that emotions are strongly influenced by an individual's conscious cognitive appraisal of a preceding situation or occurrence. ${ }^{17}$ According to this theory, ultramarathon runners with favourable appraisals of their performance should experience positive moods states after a race but runners who appraise their performance as being poor should experience negative mood states. Furthermore, the way athletes appraise their performance will perhaps in turn depend upon their underlying subjective motivations and meanings associated with the race, making the relationship between thoughts and feelings a complex one for ultramarathon runners.

The aims of this study were to measure changing patterns of mood among runners before and after an ultramarathon, and explore the relationship between the accuracy of performance expectations, perceived exertion and mood state among ultramarathon runners. It was hypothesised that runners who perform an ultramarathon better than or close to their expectations will experience more positive changes than runners whose performance is worse than expected. An additional purpose of this study was to measure the amplitude and time course of any mood disturbances experienced during the post-race recovery period.

\section{Method}

\section{Participants}

Eight male and 2 female runners were recruited from the Puffer ultramarathon entrant list. The Puffer ultramarathon is a challenging mountainous $73.4 \mathrm{~km}$ trail race that runs between Cape Point and Cape Town across Table Mountain National Park in South Africa. All of the participants were moderately or well-trained endurance runners who had completed at least one ultramarathon or Ironman triathlon as a qualifier during the 6-month period preceding the study. One female runner did not start the race and one male runner did not finish the race, leaving 8 participants in the study. The age and body mass of runners who completed the study were $41.8 \pm 7.1$ years and $72.0 \pm 8.6 \mathrm{~kg}$. Body mass was measured within 1 hour of the start of the race using portable scales. A power analysis indicated a sample size of between 8 and 12 participants would be satisfactory to achieve $p$-values of $<0.05$ and $<0.01$ respectively for a one-tailed linear correlation test with an $r$-value of $>0.7$ and power of 0.6. All runners provided their written informed consent to participate in the study, which was approved by the University of Cape Town ethics committee and conducted in accordance with the Declaration of Helsinki (amended 2008).

\section{Mood state measurements}

Throughout this study mood state was measured using the McNair, Lorr \& Droppleman $(1971,1992)$ shortened 'right now' version of the Profile of Mood States questionnaire (POMS). ${ }^{18}$ The POMS short form comprises 30 single-word mood descriptors, each with a 5point Likert response scale, from which subscale scores for tension, depression, anger, vigour, fatigue, and confusion could be calculated. The POMS short version was used to minimise questionnaire fatigue associated with the high number of POMS trials in this study, and the same investigator was used to administer all of the POMS questionnaires. To minimise response bias effects, runners were briefed to complete the POMS based upon how they felt at the time rather than attempting to memorise their previous responses, and every time a runner completed a POMS they were not able to see any previous questionnaire that they had completed.

Each participant completed a POMS 2 days before the race (baseline trial), on the morning of the race (pre-race trial), immediately after the race (post-race trial) and then at 5, 10, 15, 20, 25, 30, 40, 50, $60,75,90$ and 105 minutes during recovery. In order to counteract the potential response bias associated with repeated post-race POMS trials, participants were instructed to answer the POMS as honestly as possible by reflecting on how they felt at that precise moment in time rather than attempting to provide answers based on any previous POMS responses that they may have remembered giving.

\section{Performance expectations}

Two days prior to the race participants were asked to provide an estimate of the time they realistically expected to complete the race (performance expectancy). Each participant's official ultramarathon race time was recorded in minutes. The accuracy of each runner's performance expectations was calculated by subtracting their actual race time from their predicted race time. This calculation produced a negative number for runners who were slower than expected and a positive number for runners who were faster than expected. All of the runners were familiar with the race profile and the predicted weather conditions when they gave their performance expectations.

\section{Perceived exertion and performance measurements}

Ratings of perceived exertion (RPE) using the $6-20$ Borg scale $^{19}$ were taken at 13 discrete checkpoints throughout the race including the finish line. Elapsed times between each of the check points were recorded for each participant and then used to calculate their average running speed.

\section{Statistical analysis}

In accordance with the instruction manual, ${ }^{18}$ POMS raw scores were converted to normalised student $t$-scores for each of the six subscales and total mood disturbance scores (TMD) was calculated 


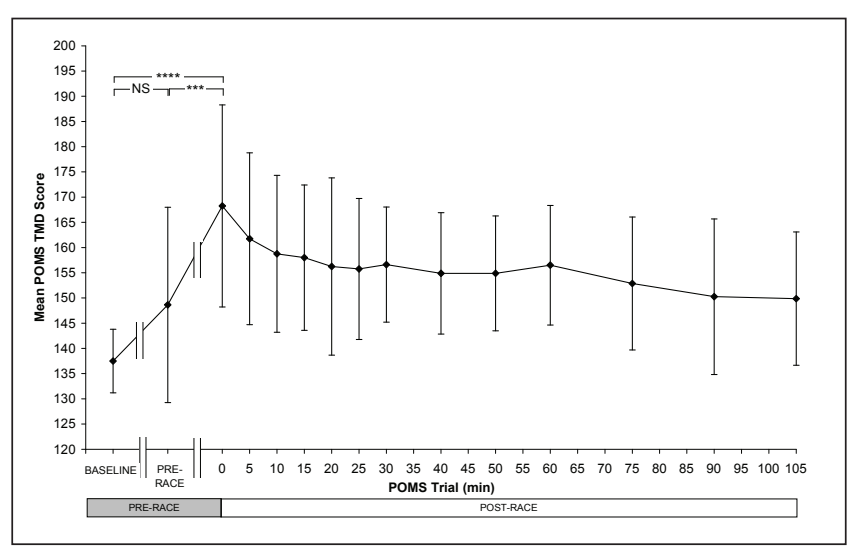

Fig. 1. Pre- and post-race changes in POMS total mood disturbance. Increases in TMD relative to baseline scores represent a negative change in mood. NS = not significant; ${ }^{* * *} p<0.005 ;{ }^{* * * *} p<0.001$.

by subtracting the POMS vigour score from the sum of tension, anger, depression, fatigue, and confusion scores.

Changes in mood state associated with the race were evaluated by comparing baseline, pre-race and post-race measures. For the POMS subscales this was determined using a MANOVA with post hoc univariate ANOVAs and paired samples $t$-tests. Trial differences in TMD were measured using a repeated-measures one-way ANOVA with post hoc paired sample $t$-tests. A Bonferonni corrected alpha level of .0167 was used to indicate statistical significance with all $t$-test results. Post-exercise changes in TMD were examined using a time series analysis with Holt's linear trend exponential smoothing to predict the length of time needed for TMD to return to baseline levels (TMD recovery).

The amplitude of pre-race changes in TMD and POMS subscales was calculated by subtracting baseline values from pre-race values. The amplitude of post-race TMD change was calculated by subtracting baseline TMD scores from post-race TMD scores. Pearson's Product Moment correlation tests were used to test relationships between the accuracy of performance expectations, average RPE score and the amplitude of post-race changes in POMS TMD. Pearson's Product Moment correlation tests were also used to measure relationships between RPE against (i) distance run and (ii) percentage of race time completed. All effect sizes are calculated as partial eta squared $\left(\eta P^{2}\right)$ or eta squared $\left(\eta^{2}\right)$.

\section{Results}

\section{Total mood disturbance (TMD)}

Repeated measures one-way ANOVA indicated a difference in TMD scores between the trials, $F(2,21)=15.2, p=0.0003, \eta P^{2}=0.69$. Post hoc paired samples $t$-tests indicated higher post-race TMD scores

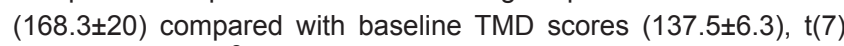
$=-5.6, p=0.001, \eta^{2}=0.82$, and compared with pre-race TMD scores (148.6 \pm 19.4$), \mathrm{t}(7)=-3.7, p=0.008, \eta^{2}=0.66$. There was no difference between baseline and pre-race TMD scores. Results of the time series analysis indicated a mean recovery time of $142 \pm 89$ min with a range of $0-265$ min. Mean TMD changes are presented in Fig. 1.

\section{Profile of mood states subscales}

A MANOVA indicated a difference in POMS subscale scores between the trials, $F(12,32)=4.6, p=0.0003, \eta_{P}^{2}=0.63$ and subsequent

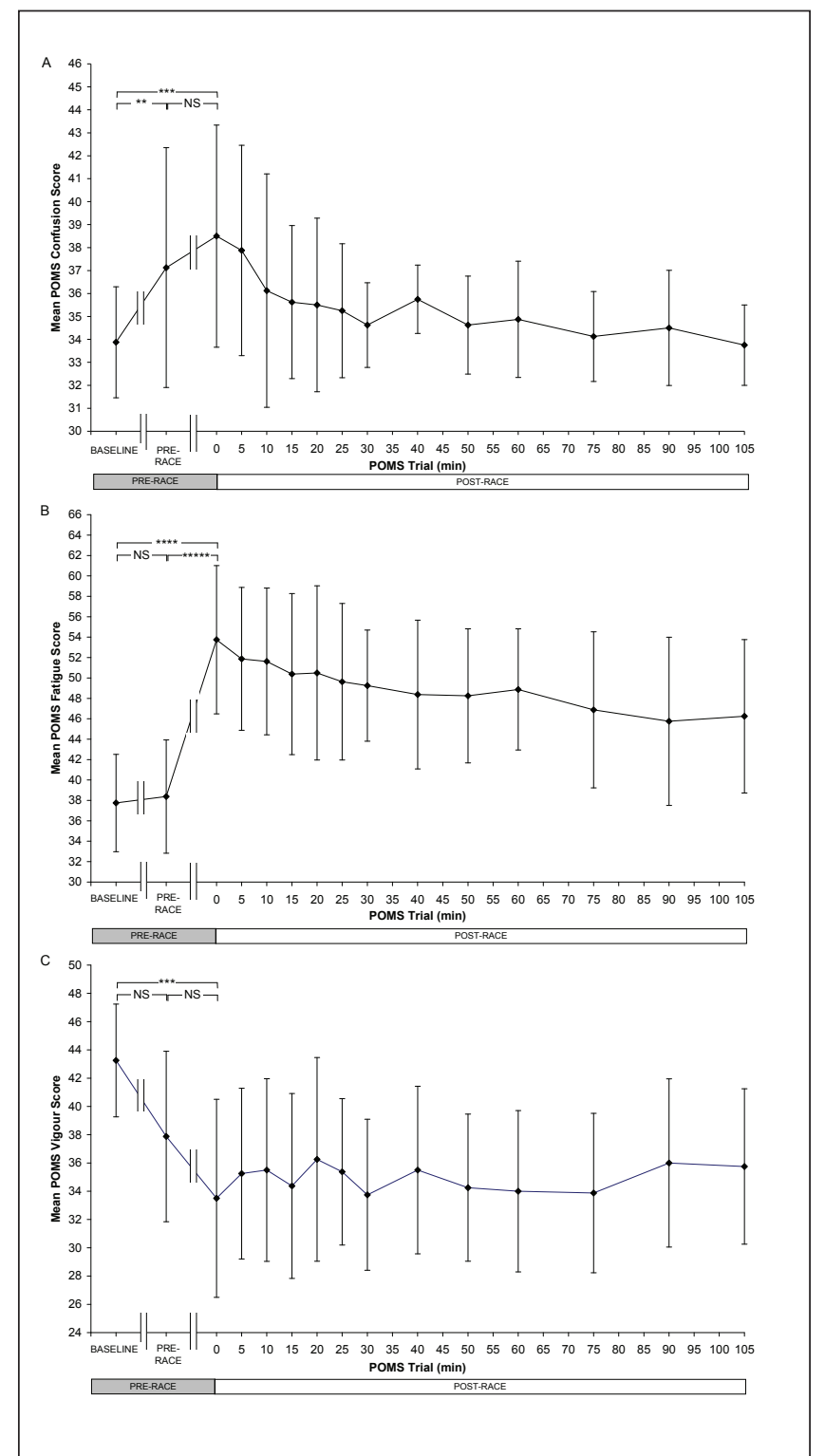

Fig. 2. Pre- and post-race changes in POMS confusion (A), fatigue (B) and vigour (C). Decreased vigour and increased confusion and fatigue relative to baseline score indicate a negative change in mood. NS = not significant; ${ }^{* *} \mathrm{p}<0.01 ;{ }^{* * *} \mathrm{p}<0.005$; $\mathrm{p}<0.001 ;{ }^{* * * *} \mathrm{p}<0.0001$

univariate ANOVAs revealed differences for the confusion subscale, $F(2,21)=3.5, p=0.05, \eta P^{2}=0.25$, the fatigue subscale, $F(2,21)=18.5$, $p=0.00002, \eta P^{2}=0.64$, and the vigour subscale, $\mathrm{F}(2,21)=5.6, p=0.011$, $\eta P^{2}=0.35$.

Post hoc paired samples $t$-tests indicated no differences between baseline and pre-race scores for POMS fatigue and vigour but an increase in confusion was found $(33.3 \pm 0.7 \mathrm{v} .37 .1 \pm 5.2), t(7)=-2.8$, $p=0.014, \eta^{2}=0.53$. An increase between baseline and post-race scores was found for POMS confusion $(33.3 \pm 0.7$ v. $38.5 \pm 4.8), t(7)=$ $-3.5, p=0.006, \eta^{2}=0.64$ and POMS fatigue (37.8 \pm 4.8 v. $\left.53.8 \pm 7.3\right)$, $\mathrm{t}(7)=-6.7, p=0.0003, \eta^{2}=0.87$. A decrease between baseline and post-race scores was found for POMS vigour ( $43.3 \pm 4.0$ v. $33.5 \pm 7.0$ ), $\mathrm{t}(7)=3.2, p=0.008, \eta^{2}=0.59$. Mean POMS subscale differences are presented in Fig. 2. 
Race performance, performance expectation accuracy, RPE and mood change

Average race completion time was 11:31:36 $\pm 00: 26: 32$ (hh:mm:ss), average running speed was $6.4 \pm 2.2 \mathrm{~km} \cdot \mathrm{hr}^{-1}$ and average RPE was 14.1 \pm 2.0 . The average running speed and RPE for each of the 13 check points is illustrated in Fig. 3A. A positive correlation was found between the distance run and RPE, $r=0.737, p=0.002$ (Fig. 3A) and between the percentage of race time completed, $r=0.725, p=0.003$ (Fig. 3B). A negative correlation was found between running speed and RPE, $r=-0.687, p=0.005$ (Fig. 3C).

Pearson's Product Moment Correlation test showed that there was no correlation between the accuracy of performance expectations and the amplitude of pre-race TMD change $(r=-0.386$, $p=0.172)$ or pre-race confusion change $(r=-0.477, p=0.116)$. A negative correlation was found between the accuracy of performance

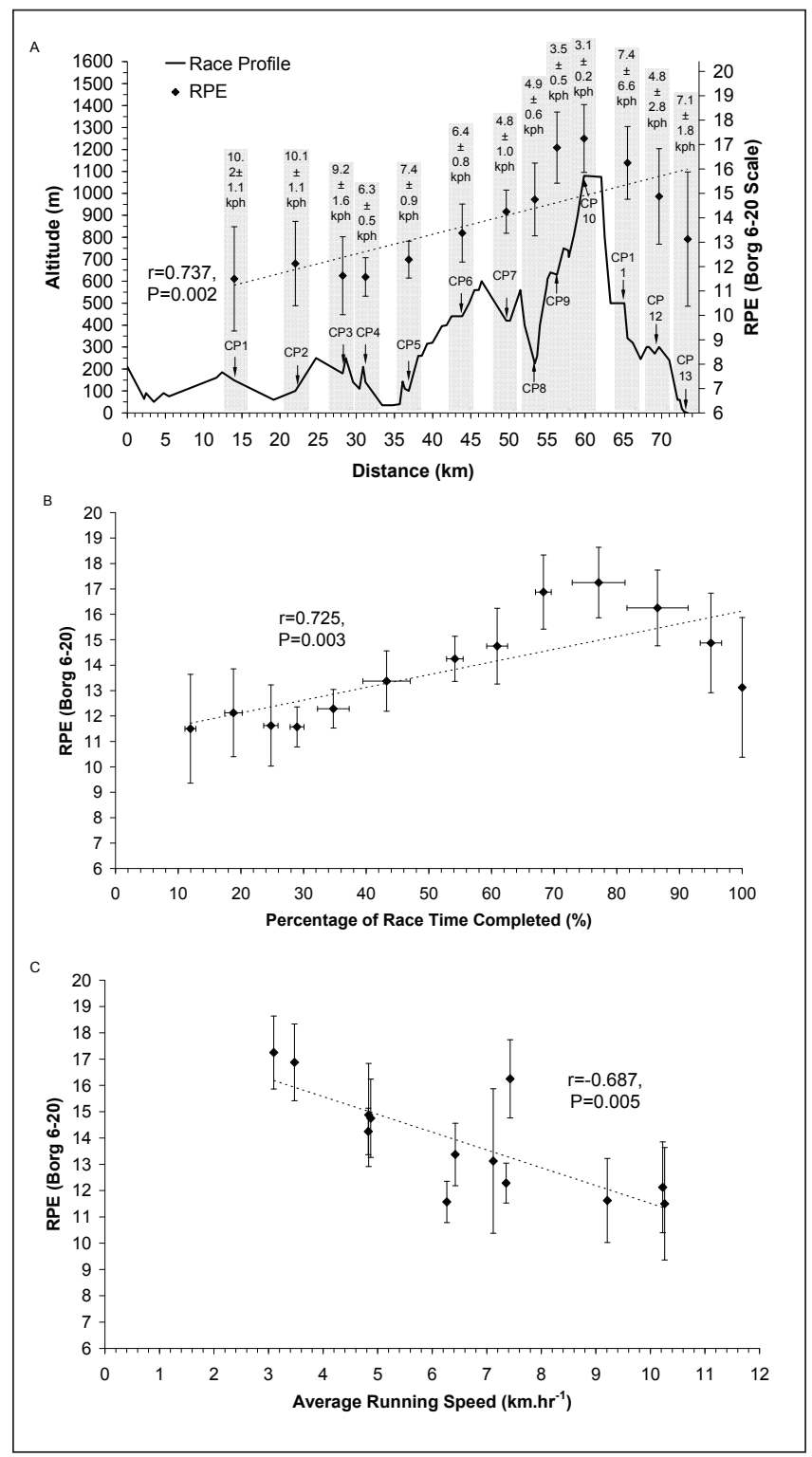

Fig. 3. Puffer ultramarathon race profile with altitude plotted on the primary $y$-axis and RPE plotted on the secondary $y$-axis (A). Relationship of RPE with percentage of race time completed (B) and average running speed (C). Y-error bars represent $\pm 1 S D$ for $R P E$ measurements; $X$-error bars represent $\pm 1 S D$ for percentage of overall race time completed at each check point. Average speed $\pm 1 S D$ between checkpoints is provided in $3 A$. expectations and post-race TMD amplitude, $r=-0.704, p=0.026$ (Fig. 4A) and post-race confusion amplitude, $r=-0.661, p=0.037$ (Fig. 4B). A positive correlation was found between the accuracy of performance expectations and post-race vigour amplitude, $r=0.840$, $p=0.005$ (Fig. 4C). There were no other correlations between the accuracy of performance expectations, average RPE, other postrace changes in mood state or TMD recovery time.

\section{Discussion}

\section{Anticipatory pre-race mood change}

The first important finding of this study was the change in POMS that occurred immediately before the race was characterised by increased levels of confusion. This suggests an anticipatory affective state that, in the absence of actual running sensations, was most likely to be a consequence of participants' conscious thoughts about their pre-race circumstances and the impending start of the race. The large standard deviation observed in confusion, and indeed all other POMS scores, reflects the usual inter-individual variation in feelings of mood but what are more important are intra-individual changes in mood compared with baseline measures. The elevated feelings of confusion experienced by runners immediately before the race may have been due to differing cognitive appraisals and interpretations regarding their specific circumstances and readiness leading up to the race. This is consistent with the view that cognitive theories are needed to account for intra- and inter-individual differences in affect. $^{20}$

In addition to increased confusion, it is surprising that participants did not experience increased feelings of tension before the race given the high stakes associated with failure and wasting months of training and preparation. Even though confusion was the only POMS subscale affected immediately before the race, the results lend some support to the general idea of mood being influenced by conscious cognitive processes. ${ }^{21}$ More specifically, it seems that an ultramarathon runner's pre-race mood state is probably strongly influenced by their own cognitive appraisals. The exact nature of these appraisals needs further investigation because in this study they were not specifically measured.

\section{Anticipatory mood state and ultramarathon perform- ance}

The increased confusion that was observed before the race did not seem to be associated with the runners' overall race performance since there was no correlation between changes in either pre-race confusion or TMD and the magnitude of their performance time prediction error. Although not measured in our study it may be that if performance is in anyway influenced by anticipatory affective states, such effects only occur at the beginning of an endurance event but gradually diminish as the race progresses and the sensory, perceptual and cognitive experience of the athlete changes. What is important is the extent to which an athlete's overall endurance event performance is influenced, if at all, by anticipatory changes in mood. Since mood is transient in nature ${ }^{20}$ it seems quite unlikely that pre-race mood states could have any significant effect on lengthy ultramarathon performances.

\section{Ultramarathon performance, RPE and post-race mood}

The linear increases in RPE that were observed when expressed relative to both distance covered and the percentage of exercise time completed suggest that, consistent with previous findings, ${ }^{22,23}$ an anticipatory strategy to prevent premature fatigue was adopted by the runners whereby they modulated running speed according to their 


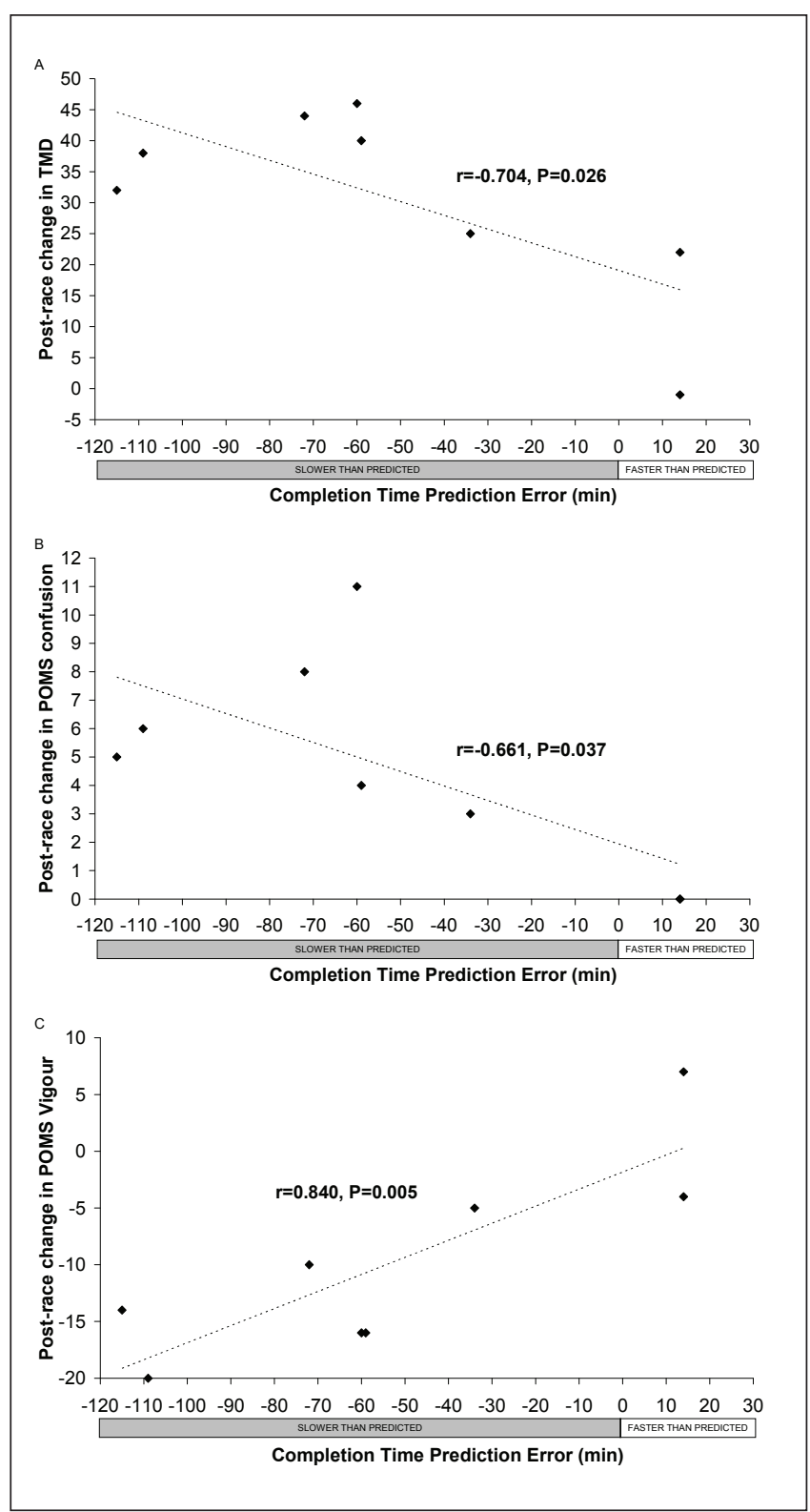

Fig. 4. Scattergrams showing the relationship between accuracy of runners' performance expectations and their post-race changes in TMD (A), POMS confusion (B) and POMS vigour (C).

feelings of exertion. All but one of the runners in this study performed worse than they predicted yet, despite the extremely mountainous terrain, all managed to complete the race by performing within sustainable RPE limits that appeared to become less conservative towards the end point as completion certainty increased. ${ }^{24}$ This suggests that, together with the inconsistent changes in average running speed at the various checkpoints, RPE is of greater importance to pacing than pursuing any performance predictions.

A large increase in TMD was observed immediately after the event that was characterised by increases in fatigue and confusion, and a reduction in vigour. Given the long and difficult nature of the race such outcomes are not particularly surprising except that, similar to pre-event changes in affect, a great deal of intra-individual variance in the magnitude of post-race affect was found that was surprisingly not strongly associated with their RPE. This suggests that the relationship between an individual's sensory experience during an endurance event and their affective response is complex and probably mediated by other psychological factors such as conscious appraisals about their performance. It is also acknowledged that from the data we collected it is not possible to differentiate between the extent to which race-specific cognitions and non-race related cognitions influenced runners' post-race mood.

What is apparent from the results is that the majority of participants ran slower than their stated performance expectations which, in addition to the physiological ramifications of running longer, potentially accounts for the unpleasant post-race changes in TMD. In fact, one of the most important findings of this study is the moderate negative correlation that was detected between runners' post-race TMD amplitude and the accuracy of their performance expectations. Most of the runners experienced unpleasant post-race mood states, the magnitude of which appears to be at least partly associated with the degree of accuracy in performance expectancy (Fig. 3). Perhaps the optimistic performance predictions made by the runners in this study was partly due to the fact that a large proportion of the Puffer ultramarathon was off-road and incorporated extreme gradients, making the prediction task more difficult, especially for those runners more accustomed to road running.

It is likely that, in endurance events, a runner's mood state at the end of a race is perhaps more a consequence of cognitive processes that develop during the race rather than RPE as they become gradually aware of whether or not they will achieve their expectations. For example, at the beginning of a long race there will still be some degree of uncertainty about the accuracy of their performance expectations but as they progress towards the end this uncertainty will become resolved according to whether predetermined checkpoints are achieved within expected times or, in instances where the athlete receives continual progression feedback, whether expected average speeds are met. Consequently, a runner's performance appraisals are likely to continually change during a race and perhaps it is these cognitive performance appraisals that have an influence on the feelings of mood experienced by runners immediately after completing the race. This is consistent with both attribution ${ }^{14}$ and appraisal theory ${ }^{16}$ in that cognition and feeling states are associated with each other. It is acknowledged that in our study no detailed record of performance appraisals were made and clearly this needs to be done in the future.

For some runners completing an ultramarathon or simply enjoying the experience might be interpreted as a success but, as in this study, others' performance appraisals might be based around achieving a specified time. Perhaps, runners' post-race mood states are also a function of appropriate goal setting, a concept that is closely dependent upon realistic performance expectations. One way in which future studies could be improved would be to collect detailed information of this sort from runners prior to their race. This would enable a better understanding of how many factors, such as previous ultramarathon experience, training status and environmental conditions, influence runners' emotional states during and after a race. Given that our study showed most runners performed worse than expected, it would appear that caution is needed when establishing endurance event outcome goals from performance predictions and expectations.

\section{Post-race mood recovery dynamics}

The amount of time needed for post-event changes in TMD to recover to baseline levels was found to be on average $2 \mathrm{hr}$ and $22 \mathrm{~min}$ but varied a great deal among participants and extended for as long as $4 \mathrm{hr}$ and $25 \mathrm{~min}$. Considering the enduring nature of an ultramarathon our findings suggest a comparatively short recovery period for mood but it is emphasised that this does not reflect participants' readiness 
to perform running or any other type of physical activity which will depend upon the recovery of a myriad of other psychological and physiological parameters. Correlation tests showed that TMD recovery time did not appear to be related to TMD amplitude or the accuracy of performance expectations. In other words, runners who experienced a big change in affect after the race or whose performance was slower than expected did not necessarily have a longer affect recovery time. Again, a more detailed investigation is needed to investigate the cognitive mediators that influence the time course of mood recovery after an ultramarathon.

\section{Conclusions}

Despite the limitations associated with low participant numbers, the findings of this study provide new insight about patterns of RPE and mood change experienced by ultramarathon runners which is a seriously under-researched area with important applied implications. For unknown reasons, endurance runners appear to make optimistic performance predictions which applied sport psychologists ought to be aware of, particularly if they intend to use these predictions as a basis for certain interventions like goal setting. Perhaps preperformance psychological interventions could focus on refining the accuracy of ultra-endurance athletes' predictions given that failing to meet performance expectations appears to exacerbate any unpleasant post-race mood states. Making sure that runners train under similar conditions in the lead-up to a race is very important in terms of developing experience and awareness of their true potential which they can drawn upon to manage and pace themselves accordingly on the day. Reinforcing race-relevant experience could be achieved through both physical and psychological training.

Although not directly measured in this study, there is some inference that cognitive performance appraisals occur throughout the race that are perhaps gradually developed and updated through repeated cycles of comparing expectations against current performance, and that it is these appraisals that underpin post-race affective experience. Further investigation is warranted to explore in more detail the relationship between explicit cognitive process and mood change among ultramarathon runners.

\section{REFERENCES}

1. Wu HJ, Chen KT, Shee BW, Chang HC, Huang YJ, Yang RS. Effects of 24 $\mathrm{h}$ ultra-marathon on biochemical and hematological parameters. World $\mathrm{J}$ Gastroenterol 2004;10(18):2711-2714.

2. Millet GY, Lepers R, Maffiuletti NA, Babault N, Martin V, Lattier G. Alterations of neuromuscular function after an ultramarathon. J App Physiol 2002;92:486-492.

3. Strachan AF, Noakes TD, Kotzenberg G, Nel AE, De Beer FC. C reactive protein concentrations during long distance running. Br Med J (Clin Res Ed) $1984 ; 289(6454): 1249-1251$.
4. Davies CT, Thompson MW. Physiological responses to prolonged exercise in ultramarathon athletes. J App Physiol 1986;61:611-617.

5. Folkins $C$, Wieselberg-Bell N. Personality profile of ultramarathon runners: a little deviance may go a long way. J Sport Behavior 1981;4(3):119-127.

6. Utter AC, Kang J, Nieman DC, Vinci DM, et al. Ratings of perceived exertion throughout an ultramarathon during carbohydrate ingestion. Perceptual and Motor Skills 2003;97(1):175-184.

7. Rauch TM, Tharion WJ, Strowman SR, Shukitt BL. Psychological factors associated with performance in the ultramarathon. J Sports Med Physical Fitness 1988;28(3):237-246.

8. Weinberg R, Jackson A, Kolodny K. The relationship of massage and exercise to mood enhancement. The Sport Psychol 1988;2:202-211.

9. Dyer JB, Grouch JG. Effects of running and other activities on moods. Percept Motor Skills 1988;67(1):43-50.

10. Markoff RA, Ryan P, Young T. Endorphins and mood changes in long-distance running. Med Sci Sports Exercise 1982;14(1):11-15.

11. Hassmén P, Blomstrand E. Mood change and marathon running: A pilot study using a Swedish version of the POMS test. Scand J Psychol 1991;32:225-232.

12. Yates A, Shisslak CM, Allender J, Crago M, Leehey K. Comparing obligatory to nonobligatory runners. Psychosomatics 1992;33:180-189.

13. Galper DI, Trivedi MH, Barlow CE, Dunn AL, Kampert JB. Inverse Association between Physical Inactivity and Mental Health in Men and Women. Med Sci Sports Exercise 2006;38(1):173-178.

14. Tharion WJ, McMenemy DJ, Terry AL, Rauch TM. Recovery of mood changes experienced when running and ultramarathon. Perceptual and Motor Skills 1990;71:1311-1316.

15. Weiner B. An attributional theory of motivation and emotion. New York: Springer, 1986.

16. Lazarus RS. Emotion and Adaptation. New York: Oxford University Press, 1991.

17. Scherer KR. Emotion as a process: function, origin, and regulation. Social Sci Info 1982;21:555-570.

18. McNair DM, Lorr M, Droppleman LF. Profile of mood state manual (revised). San Diego, CA: Educational and Industrial Testing Service, 1992.

19. Borg G. Psychophysical bases of perceived exertion. Med Sci Sports Exercise 1982;14:377-381.

20. Power M, Dalgleish T. Cognition and emotion: from order to disorder. Hove: Psychology Press, 1997.

21. Riskind $\mathrm{JH}$. The mediating mechanisms in mood and memory: a cognitive priming formulation. In D. Kuiken (ed.), Mood and memory: theory, research and applications. Special issue of J Social Behav Personality 1989;4:39-43.

22. Crewe H, Tucker R, Noakes TD. The rate of increase in perceived exertion predicts the duration of exercise to fatigue at a fixed power output in different environmental conditions. Eur J Appl Physiol 2008;103:569577.

23. Noakes TD, Snow RJ, Febbraio MA. Letter to the editor. J Appl Physiol 2003;96:1571-1573.

24. Tucker R, Noakes T. The anticipatory regulation of performance: The physiological basis for pacing strategies and the development of a perception-based model for exercise performance. $\mathrm{Br} \mathrm{J}$ Sports Med 2009;43(6):392-400. 